\title{
ON THE CONVERGENCE OF FULLY-DISCRETE HIGH-RESOLUTION SCHEMES WITH VAN LEER'S FLUX LIMITER FOR CONSERVATION LAWS*
}

\author{
NAN JIANG ${ }^{\dagger}$
}

\begin{abstract}
A class of fully-discrete high-resolution schemes using flux limiters was constructed by P. K. Sweby [SIAM J. Numer. Anal. 21 (1984), 995-1011], which amounted to add a limited anti-diffusive flux to a first order scheme. This technique has been very successful in obtaining high-resolution, second order, oscillation free, explicit difference schemes. However, the entropy convergence of such schemes has been open. For the scalar convex conservation laws, we use one of Yang's convergence criteria [SIAM. J. Numer. Anal. 36 (1999) No. 1, 1-31] to show the entropy convergence of the schemes with van Leer's flux limiter when the building block of the schemes is the Godunov or the Engquish-Osher. The entropy convergence of the corresponding problems in semi-discrete case, for convex conservation laws with or without a source term, has been settled by Jiang and Yang [Methods and Applications of Analysis 12 (2005), No. 1, 089-102].
\end{abstract}

Key words. Conservation law, fully-discrete schemes with flux limiters, entropy convergence.

AMS subject classifications. 65M12 (35L60)

1. Introduction. We consider numerical approximations to the scalar conservation laws

$$
\left\{\begin{array}{l}
u_{t}+f(u)_{x}=0, \\
u(x, 0)=u_{0}(x),
\end{array}\right.
$$

where $f \in C^{1}(\mathbb{R})$ is convex, and $u_{0} \in B V(\mathbb{R})$. Here $B V$ stands for the subspace of $L_{l o c}^{1}$ consisting of functions $z$ with bounded total variation

$$
T V(z):=\sup _{h \neq 0} \int_{\mathbb{R}} \frac{|z(x+h)-z(x)|}{|h|} d x .
$$

In particular, we are interested in numerical schemes that admit conservative form

$$
u_{k}^{n+1}=H\left(u_{k-p}^{n}, \cdots, u_{k+p}^{n} ; \lambda\right)=u_{k}^{n}-\lambda\left(g_{k+\frac{1}{2}}^{n}-g_{k-\frac{1}{2}}^{n}\right),
$$

where $h$ and $\tau$ are spatial and temporal steps respectively, $\lambda=\frac{\tau}{h} ; u_{k}^{n}=u\left(x_{k}, t_{n}\right)$ are nodal values of the piecewise constant mesh function $u_{h}(x, t)$ approximating the solution $u(x, t)$. The numerical flux $g$ is given by

$$
g_{k+\frac{1}{2}}^{n}=g_{k+\frac{1}{2}}\left[u^{n} ; \lambda\right]
$$

where

$$
g_{k+\frac{1}{2}}[v ; \lambda]=g\left(v_{k-p+1}, v_{k-p+2}, \cdots, v_{k}, \cdots, v_{k+p} ; \lambda\right),
$$

for any data $\left\{v_{j}\right\}$. Throughout the paper, we simply write $g_{k+\frac{1}{2}}[v ; \lambda]$ as $g_{k+\frac{1}{2}}[v]$ whenever this is not ambiguous.

\footnotetext{
*Received September 21, 2009; accepted for publication December 22, 2009.

${ }^{\dagger}$ Department of Mathematical Sciences of University of South Dakota, Vermillion SD 57069, USA (njiang@usd.edu).
} 
The function $g$ is Lipschitz continuous with respect to its first $2 p$ arguments and is consistent with the conservation law in the sense that

$$
g(u, u, \cdots, u, \lambda) \equiv f(u) .
$$

A scheme of the form (1.3)-(1.6) is called self-similar if $\lambda$ is fixed, i.e., $g$ is independent of the step-size. In this paper, we only consider self-similar schemes, which contain many classical high-resolution schemes. We would mention that, for the entropy convergence analysis, it is in the case of self-similar schemes that one faces the most formidable challenge. See [15] for a comment on entropy analysis of schemes with step-size dependent fluxes. The collection of points $\left\{x_{k-p}, \cdots, x_{k+p}\right\}$ is said to be the stencil of the scheme at the point $\left(x_{k}, t_{n}\right)$. For a sequence of numerical solutions, we assume that the corresponding sequence of step sizes tends to zero.

Initial ideas of using flux limiters to construct high-resolution schemes can be traced back, for example, to the early works of van Leer [14], Roe [10] and, Chakravarthy and Osher [1]. Later, Sweby's contributions along this direction [12] include: introducing a class of limiters, unifying the flux limiters independently studied by van Leer, Roe and, Chakravarthy and Osher into his general framework, presenting a technique to obtain a scheme that is high-resolution, second order and oscillation free by the addition of a limited anti-diffusive flux to a first order scheme, and investigating the entropy convergence of these schemes. His preliminary analysis has supported his conjecture of the entropy satisfaction of any such scheme, provided that the underlying first order scheme (which we called the building block of the scheme) is entropy satisfying and diffusion at expansions is not decreased with respect to its building block. Although, the schemes with flux limiters have been demonstrated to be some of the most effective methods that are capable of producing high-resolution shock profiles (see, for example, $[9,12]$ ), the rigorously theoretical analysis of the entropy consistence of these schemes has been fallen behind. In fact, the convergence issues of the fully-discrete flux limiter schemes, introduced by Sweby, remain open. The objective of this paper is to settle one of these open problems.

During the 80's, the entropy convergence analysis heavily depends on the classical cell entropy inequality (CEI) approach, which demands to establish entropy inequality for each computational cell. As a result, the entropy convergence of many effective schemes, such as flux limiter schemes, cannot be justified by CEI method. Recent years, among the different approaches emerged, Yang's wavewise entropy inequality (WEI) $[15,16]$ concept has been stand out. For semi-discrete and fully-discrete schemes, this new concept has produced four convergence criteria respectively. For convex conservation laws, one of criteria, in semi- or fully-discrete case, essentially states that, a WEI across the area of rarefaction where $u_{j} \leq u_{j+1}$ for all $x_{j}$ is sufficient for convergence to the entropy solution. Hence, in the convergence analysis, one may safely remove the shock area from scrutiny. Further, even in the rarefaction area, a much weaker condition than CEI is sufficient for the convergence. To demonstrate that this approach has brought a powerful tool into the success of convergence analysis, we name the following established convergence results.

In the semi-discrete case, for homogeneous convex conservation laws, Yang [15] has shown the entropy convergence of the generalized MUSCL scheme and the schemes based on minmod limiter [9] when the general building block of the schemes is an arbitrary $E$-scheme [8], and based on Chakravarthy-Osher limiter [1] when the building block of the schemes is the Godunov [3], the Engquist-Osher [2], or the Lax-Friedrichs [7]. Recently, Yang and the author $[5,17]$ have made significant advances at this front. 
Not only we have extended all of previous convergence results of Yang to the convex conservation laws with an arbitrary $C^{1}$ source term, we have further established that, for convex conservation laws with or without a source term, the schemes with van Leer's limiter $[12,14]$, when the building block of the schemes is the Godunov, the Engquist-Osher, or the Lax-Friedrichs, converge to the physically correct solution as well.

In the fully-discrete case, the convergence analysis is generally more challenge, yet for homogeneous convex conservation laws, Yang [16] has shown the entropy convergence of explicit MUSCL scheme using his forth convergence criterion. The main result of this paper is the entropy convergence of the schemes with van Leer's limiter when the building block of the schemes is the Godunov or the Engquist-Osher. Again, we use Yang's last convergence criterion of the fully-discrete case to show the convergence.

The paper is organized as follows. In section 2, we first review the notions of the extremum paths and the schemes with flux limiters. We then establish the extremum traceableness of the general TVD (total variation diminishing) schemes, which is under the necessity of analyzing the entropy convergence that follows in the next section. In section 3, we present a simplified version of Yang's convergence criterion, an important estimate, and the proof of the main result.

\section{Extremum traceableness of the TVD schemes.}

2.1. The extremum paths. In this subsection, we review the flux limiter methods described by Sweby [12] and the notions of Yang's extremum paths [16]. For the consistency, we closely follow notations introduced by Sweby and Yang respectively. Denote

$$
\begin{gathered}
\left(\Delta f_{k+\frac{1}{2}}\right)^{+}=f\left(u_{k+1}^{n}\right)-g_{k+\frac{1}{2}}^{E} \\
\left(\Delta f_{k+\frac{1}{2}}\right)^{-}=g_{k+\frac{1}{2}}^{E}-f\left(u_{k}^{n}\right)
\end{gathered}
$$

where $g_{k+\frac{1}{2}}^{E}=g^{E}\left(u_{k}^{n}, u_{k+1}^{n}\right)$ is the flux of an $E$-scheme [8] that satisfies

$$
\operatorname{sgn}\left(u_{k+1}^{n}-u_{k}^{n}\right)\left[g_{k+\frac{1}{2}}^{E}-f(u)\right] \leq 0
$$

for all $u$ in between $u_{k}^{n}$ and $u_{k+1}^{n}$.

We use

$$
\nu_{k+\frac{1}{2}}^{+}=\frac{\lambda\left(\Delta f_{k+\frac{1}{2}}\right)^{+}}{\Delta u_{k+\frac{1}{2}}^{n}}, \quad \nu_{k+\frac{1}{2}}^{-}=\frac{\lambda\left(\Delta f_{k+\frac{1}{2}}\right)^{-}}{\Delta u_{k+\frac{1}{2}}^{n}}
$$

to define a series of local CFL numbers, where, by convention, $\Delta u_{k+\frac{1}{2}}^{n}=\Delta_{+} u_{k}^{n}=$ $\Delta_{-} u_{k+1}^{n}=u_{k+1}^{n}-u_{k}^{n}$. We also set

$$
\alpha_{k+\frac{1}{2}}^{+}=\frac{1}{2}\left(1-\nu_{k+\frac{1}{2}}^{+}\right), \quad \alpha_{k+\frac{1}{2}}^{-}=\frac{1}{2}\left(1+\nu_{k+\frac{1}{2}}^{-}\right)
$$


and

$$
r_{k}^{+}=\frac{\alpha_{k-\frac{1}{2}}^{+}\left(\Delta f_{k-\frac{1}{2}}\right)^{+}}{\alpha_{k+\frac{1}{2}}^{+}\left(\Delta f_{k+\frac{1}{2}}\right)^{+}}, \quad r_{k}^{-}=\frac{\alpha_{k+\frac{1}{2}}^{-}\left(\Delta f_{k+\frac{1}{2}}\right)^{-}}{\alpha_{k-\frac{1}{2}}^{-}\left(\Delta f_{k-\frac{1}{2}}\right)^{-}} .
$$

Very often, to enhance the readability, we use Sweby's shorthand notations: $u^{k} \equiv$ $u_{k}^{n+1}, u_{k} \equiv u_{k}^{n}$, where $k$ and $n$ are the spatial and temporal indices respectively. The schemes with flux limiter are given by

$$
u^{k}=u_{k}-\lambda\left(g_{k+\frac{1}{2}}-g_{k-\frac{1}{2}}\right),
$$

where

$$
g_{k+\frac{1}{2}}=g_{k+\frac{1}{2}}^{E}+\varphi\left(r_{k}^{+}\right) \alpha_{k+\frac{1}{2}}^{+}\left(\Delta f_{k+\frac{1}{2}}\right)^{+}-\varphi\left(r_{k+1}^{-}\right) \alpha_{k+\frac{1}{2}}^{-}\left(\Delta f_{k+\frac{1}{2}}\right)^{-},
$$

and $\varphi$ is a flux limiter, which is Lipschitz continuous function and its graph lies in the second order TVD region derived by Sweby [12]:

$$
\left\{\left(r, \varphi_{\Phi}(r)\right): \varphi_{\Phi}(r)=\max (0, \min (\Phi r, 1), \min (r, \Phi)), 1 \leq \Phi \leq 2, r \in \mathbb{R}\right\} .
$$

Some of the well known instances of such flux limiters are van Leer, Roe and Chakravarthy-Osher's limiters [12]. We shall assume for the remainder of the paper that the local CFL numbers satisfy $\left|\nu_{k+\frac{1}{2}}^{ \pm}\right| \leq 1$ for all $k \in \mathbb{Z}$.

It is easy to see that the schemes (2.7)-(2.8) can be written in an increment form

$$
u^{k}=u_{k}-C_{k-\frac{1}{2}} \Delta u_{k-\frac{1}{2}}+D_{k+\frac{1}{2}} \Delta u_{k+\frac{1}{2}}
$$

with

$$
C_{k+\frac{1}{2}}=\nu_{k+\frac{1}{2}}^{+}\left\{1+\alpha_{k+\frac{1}{2}}^{+}\left[\frac{\varphi\left(r_{k+1}^{+}\right)}{r_{k+1}^{+}}-\varphi\left(r_{k}^{+}\right)\right]\right\}
$$

and

$$
D_{k+\frac{1}{2}}=-\nu_{k+\frac{1}{2}}^{-}\left\{1+\alpha_{k+\frac{1}{2}}^{-}\left[\frac{\varphi\left(r_{k}^{-}\right)}{r_{k}^{-}}-\varphi\left(r_{k+1}^{-}\right)\right]\right\} .
$$

The concept of discrete extremum paths was introduced by Yang (see Definition 6.3 [15] and Definition 2.13 [16]). For the convenience of applications, we quote relevant definitions of the fully-discrete case. Consider a numerical solution $u$ defined on the set of grid points $X:=\left\{\left(x_{j}, t_{n}\right): j \in \mathbb{Z}, n \in \mathbb{Z}^{+}\right\}$. A finite set of successive grid points $\left\{x_{q}, \cdots, x_{r}\right\}$ with $r \geq q$ is said to be the stencil of a spatial maximum, or simply an $\bar{E}$-stencil of $u$ at the time $t_{n}$, provided $u_{q}^{n}=\cdots=u_{r}^{n}, u_{q-1}^{n}<u_{q}^{n}$ and $u_{r+1}^{n}<u_{r}^{n}$. Notions of $\underline{E}$-stencils for minima and E-stencils for general extrema are defined similarly. Throughout the paper, we refer to [16] for the definitions, lemmas and theorems that we have quoted.

Definition 2.1 (see Definition 2.13 [16]). A nonempty subset of $X$ denoted by $\bar{E}_{t_{n}, t_{m}}, n \leq m$, is called a ridge of the numerical solution $u$ from $t_{n}$ to $t_{m}$ if

(i) for all $\nu, n \leq \nu \leq m$, the set

$$
P_{\bar{E}}(\nu):=\left\{x_{j}:\left(x_{j}, t_{\nu}\right) \in \bar{E}_{t_{n}, t_{m}}\right\}=\left\{x_{q^{\nu}}, \cdots, x_{r^{\nu}}\right\}
$$


is not empty and is an $\bar{E}$-stencil of $u$ at $t_{\nu}$;

(ii) for all $\nu, n \leq \nu \leq m-1$,

$$
P_{\bar{E}}(\nu) \cup P_{\bar{E}}(\nu+1)=\left\{x_{j}: \min \left(q^{\nu}, q^{\nu+1}\right) \leq j \leq \max \left(r^{\nu}, r^{\nu+1}\right)\right\} .
$$

The set $P_{\bar{E}}(\nu)$ is called the $x$-projection of $\bar{E}_{t_{n}, t_{m}}$ at $t_{\nu}$. The value of $u$ along the ridge is denoted by $V_{\bar{E}}(\nu): V_{\bar{E}}(\nu)=u_{j}^{\nu}$ for $q^{\nu} \leq j \leq r^{\nu}$.

If, for all $\nu, n \leq \nu \leq m$, the $\bar{E}$-stencil in the item (i) of the definition is replaced by an $\underline{E}$-stencil, then the set is called a trough of $u$ from $t_{n}$ to $t_{m}$ and is denoted by $\underline{E}_{t_{n}, t_{m}}$. The related notions $P_{\underline{E}}(\nu)$ and $V_{\underline{E}}(\nu)$ are defined similarly. Ridges and troughs are also called extremum paths. When we do not distinguish between ridges and troughs, we use $E_{t_{n}, t_{m}}, P_{E}(\nu)$, and $V_{E}(\nu)$ for either type. We write

$$
E_{t_{n}, t_{m}}^{1}<(\leq) E_{t_{n}, t_{m}}^{2} \text {, if } \max P_{E^{1}}(\nu)<(\leq) \max P_{E^{2}}(\nu) \text { for } n \leq \nu \leq m \text {. }
$$

Definition 2.2 (see Definition $2.14[16]$ ). A scheme is said to be extremum traceable if there exists a positive constant $c \geq 1$ such that for each numerical solution $u$ of the scheme and each integer $N>0$, there exists a finite or infinite collection of extremum paths $\left\{E_{t_{0}, t_{N}}^{l}\right\}_{l=l_{1}}^{l_{2}}$ with the following properties:

(i) $\left\{P_{E^{l}}(N)\right\}_{l=l_{1}}^{l_{2}}$ is precisely the set of $E$-stencils of $u_{j}^{n}$ at the time $t_{N}$ arranged in ascending spatial coordinates.

(ii) If $E_{t_{0}, t_{N}}^{l}$ is a ridge (trough), then $V_{E_{l}}(n)$ is a non increasing (non decreasing) function of $n$. then

(iii) Let $P_{E^{l}}(n)=\left\{x_{q^{l}(n)}, \cdots, x_{r^{l}(n)}\right\}$ for $1 \leq n \leq N$. If $P_{E^{l}}(n) \cap P_{E^{l}}(n+1)=\emptyset$,

$$
\begin{aligned}
& \left|u_{q^{l}(n+1)}^{n}-u_{r^{l}(n)}^{n}\right| \leq c\left|V_{E^{l}}(n+1)-V_{E^{l}}(n)\right| \text { when } \quad q^{l}(n+1)>r^{l}(n), \\
& \left|u_{r^{l}(n+1)}^{n}-u_{q^{l}(n)}^{n}\right| \leq c\left|V_{E^{l}}(n+1)-V_{E^{l}}(n)\right| \text { when } \quad q^{l}(n)>r^{l}(n+1) .
\end{aligned}
$$

(iv) If $l_{2}>l_{1}$, then $E_{t_{0}, t_{N}}^{l}<E_{t_{0}, t_{N}}^{l+1}$ for $l_{1} \leq l \leq l_{2}-1$.

2.2. Extremum traceableness of the TVD schemes. For the numerical solutions concerned, total variation diminishing in time is a desirable property that is shared with the exact solution of (1.1). TVD schemes prevent new extrema values (that generate spurious oscillations of the solutions) other than those which propagate from the previous time-level. In compliance with the extremum traceable condition of Yang's WEI convergence criterion, we focus our attention on the general TVD schemes and we are able to show the following result.

TheOrem 2.3. The sufficient conditions for the schemes (2.7)-(2.8) to be extremum traceable are the following inequalities:

$$
0 \leq C_{k+\frac{1}{2}}, \quad 0 \leq D_{k+\frac{1}{2}}, \quad 0 \leq C_{k+\frac{1}{2}}+D_{k+\frac{1}{2}} \leq 1, \text { for all } k ;
$$

there is a positive constant $\mu$ such that, if $u_{k}$ is a space extremum, then

$$
\max \left\{C_{k \pm \frac{1}{2}}, C_{k \pm \frac{3}{2}}, D_{k \pm \frac{1}{2}}, D_{k+\frac{3}{2}}\right\} \leq \frac{\mu}{4}<\frac{1}{4},
$$

where $C_{k+\frac{1}{2}}$ and $D_{k+\frac{1}{2}}$ are given by $(2.10)-(2.12)$. 
Notice that the inequalities of (2.13) are the Harten's sufficient TVD conditions [4] for the schemes (2.7)-(2.8). By Lax-Wendroff theorem [6] and Helly's theorem, the numerical solutions of the schemes (2.7)-(2.8) converge to a weak solution of (1.1). We will show the entropy convergence of the schemes of (2.7)-(2.8) in the next section.

Proof of Theorem 2.3. To show the extremum traceableness of a scheme of the form (2.7)-(2.8), it suffices to consider the case $N=n+1$ and to show that we can construct a collection of two level extremum paths $\left\{E_{t_{n}, t_{n+1}}^{l}\right\}_{l=l_{1}}^{l_{2}}$ that satisfies Definition 2.2.

Step 1. In this step, we show that, under the given conditions, certain patterns of two level extremum paths can not exist, which will ensure the construction of the extremum paths in Step 2, the order preserving property of the extremum paths and the backward traceability of the extrema of the scheme. We only give the proof of the case that $u^{k}$ is a maximum. When $u^{k}$ is a minimum, the proof is similar and has been omitted.

We begin with showing the first implication: if $u^{k}>u^{k \pm 1}$, and $u_{k} \leq u_{k \pm 1}$, then either $u_{k+2}<u_{k+1}$ or $u_{k-2}<u_{k-1}$. Otherwise, it derives a contradiction:

$$
\begin{aligned}
\left(u^{k}-u^{k+1}\right)+\left(u^{k}-u^{k-1}\right) & =\left(1-2 C_{k-\frac{1}{2}}-D_{k-\frac{1}{2}}\right) \Delta u_{k-\frac{1}{2}}+C_{k-\frac{3}{2}} \Delta u_{k-\frac{3}{2}} \\
& -\left(1-C_{k+\frac{1}{2}}-2 D_{k+\frac{1}{2}}\right) \Delta u_{k+\frac{1}{2}}-D_{k+\frac{3}{2}} \Delta u_{k+\frac{3}{2}} \\
& \leq 0
\end{aligned}
$$

Equivalently, if $u^{k}>u^{k \pm 1}, u_{k+2} \geq u_{k+1}$ and $u_{k-2} \geq u_{k-1}$, then $u_{k}>u_{k+1}$ or $u_{k}>$ $u_{k-1}$. In fact, by (2.13), we have the second implication: if $u^{k}>u^{k \pm 1}, u_{k+2} \geq u_{k+1}$ and $u_{k-2} \geq u_{k-1}$, then $u_{k}>u_{k \pm 1}$.

Next, we show the third implication: if $u^{k}>u^{k \pm 1}, u^{k+2}>u^{k+1}, u_{k} \leq u_{k \pm 1}$ and $u_{k+2} \leq u_{k+1}$, then either $u_{k+2}<u_{k+3}$ or $u_{k-2}<u_{k-1}$. Otherwise,

$$
\begin{aligned}
& \left(u^{k}-u^{k+1}\right)+\left(u^{k+2}-u^{k+1}\right)+\left(u^{k}-u^{k-1}\right) \\
= & -\left(1-2 C_{k+\frac{1}{2}}-2 D_{k+\frac{1}{2}}\right) \Delta u_{k+\frac{1}{2}}+\left(1-C_{k+\frac{3}{2}}-2 D_{k+\frac{3}{2}}\right) \Delta u_{k+\frac{3}{2}} \\
+ & \left(1-2 C_{k-\frac{1}{2}}-D_{k-\frac{1}{2}}\right) \Delta u_{k-\frac{1}{2}}+D_{k+\frac{5}{2}} \Delta u_{k+\frac{5}{2}}+C_{k-\frac{3}{2}} \Delta u_{k-\frac{3}{2}} \\
\leq & 0
\end{aligned}
$$

which derives a contradiction.

Finally, we show the fourth implication: if $u^{k}>u^{k \pm 1}, u_{k} \leq u_{k \pm 1}$ and $u_{k+2} \leq$ $u_{k+1}$, then $u_{k+1}-u_{k}<\max \left(u_{k-1}-u_{k}, u_{k+1}-u_{k+2}\right)$. Otherwise,

$$
\begin{aligned}
& u^{k+1}-u^{k} \\
= & \left(1-C_{k+\frac{1}{2}}-D_{k+\frac{1}{2}}\right) \Delta u_{k+\frac{1}{2}}+D_{k+\frac{3}{2}} \Delta u_{k+\frac{3}{2}}+C_{k-\frac{1}{2}} \Delta u_{k-\frac{1}{2}} \\
\geq & \left(1-C_{k+\frac{1}{2}}-D_{k+\frac{1}{2}}-D_{k+\frac{3}{2}}-C_{k-\frac{1}{2}}\right) \Delta u_{k+\frac{1}{2}} \\
\geq & 0,
\end{aligned}
$$

which gives a contradiction.

Step 2. In this step, we construct the extremum paths. Without loss of generality, we only give the construction of a ridge in the generic case. Note that the first and the second implications and Harten's TVD conditions of (2.13) guarantee that if, at time $t=t_{n+1}$,

$$
u_{q}^{n+1}>\max \left(u_{q-1}^{n+1}, u_{q+1}^{n+1}\right),
$$


then there exists at least one $\bar{E}$-stencil, at time $t=t_{n}$, among

$$
\left\{x_{q-2}, x_{q-1}, x_{q}, x_{q+1}, x_{q+2}\right\} .
$$

Let $\left\{x_{q_{\nu}^{\prime}}, \cdots, x_{r_{\nu}^{\prime}}\right\}_{\nu=1}^{\mu}$ be the set of all such $\bar{E}$-stencils at $t=t_{n}$ arranged in ascending spatial coordinates. The construction of a ridge $\bar{E}_{t_{n}, t_{n+1}}$ with $P_{\bar{E}}(n+1)=$ $\left\{x_{q}, \cdots, x_{r}\right\}$ goes as follows.

Case 1. There exists an integer $\nu$ with $1 \leq \nu \leq \mu$ such that

$$
\left\{x_{q_{\nu}^{\prime}}, \cdots, x_{r_{\nu}^{\prime}}\right\} \cap\left\{x_{q}, \cdots, x_{r}\right\} \neq \emptyset .
$$

Set $P_{\bar{E}}(n)=\left\{x_{q_{\nu}^{\prime}}, \cdots, x_{r_{\nu}^{\prime}}\right\}$ for an arbitrary $\nu$ that satisfies (2.15).

Case 2. $\mu=1,\left\{x_{q_{q_{1}}^{\prime}}, \cdots, x_{r_{r_{1}}^{\prime}}\right\}=\left\{x_{q-1}\right\}$. Set $P_{\bar{E}}(n)=\left\{x_{q-1}\right\}$.

Case 3. $\mu=1,\left\{x_{q_{q_{1}}^{\prime}}, \cdots, x_{r_{r_{1}}^{\prime}}\right\}=\left\{x_{r+1}\right\}$. Set $P_{\bar{E}}(n)=\left\{x_{r+1}\right\}$.

Case 4. $\mu=2,\left\{x_{q_{q_{1}}^{\prime}}^{\prime}, \cdots, x_{r_{r_{1}}^{\prime}}\right\}=\left\{x_{q-1}\right\},\left\{x_{q_{q_{2}}^{\prime}}, \cdots, x_{r_{r_{2}}^{\prime}}\right\}=\left\{x_{r+1}\right\}$. Set

$$
P_{\bar{E}}(n)=\left\{\begin{array}{lll}
\left\{x_{q-1}\right\} & \text { if } & u_{r+1}^{n} \leq u_{q-1}^{n} \\
\left\{x_{r+1}\right\} & \text { if } & u_{r+1}^{n}>u_{q-1}^{n}
\end{array}\right.
$$

We have exhausted all possibilities. Indeed, if

$$
\mu=2,\left\{x_{q_{q 1}^{\prime}}, \cdots, x_{r_{r 1}^{\prime}}\right\}=\left\{x_{q-2}\right\},\left\{x_{q_{q 2}^{\prime}}, \cdots, x_{r_{r 2}^{\prime}}\right\}=\left\{x_{r+2}\right\},
$$

then, by the definition of the ridge, this case implies that $u_{q-2}>u_{q-1}$ and $u_{q+2}>$ $u_{q+1}$ (i.e. $u_{r+2}>u_{r+1}$ ). Now following the second implication in the Step 1, we have $u_{q} \geq u_{q \pm 1}$, which means that this case falls into Case 1 of the construction. We can also exclude the following two cases. If

$$
\mu=1,\left\{x_{q_{q_{1}}^{\prime}}, \cdots, x_{r_{r_{1}}^{\prime}}\right\}=\left\{x_{q-2}\right\}
$$

then, by the definition of the ridge, we have $u_{q-2}^{n}>u_{q-1}^{n} \geq u_{q}^{n} \geq u_{q+1}^{n} \geq u_{q+2}^{n}$, which derives the following contradiction:

$$
\begin{aligned}
0 & <u_{q}^{n+1}-u_{q-1}^{n+1} \\
& =\left(1-C_{q-\frac{1}{2}}-D_{q-\frac{1}{2}}\right) \Delta u_{q-\frac{1}{2}}^{n}+D_{q+\frac{1}{2}} \Delta u_{q+\frac{1}{2}}^{n}+C_{q-\frac{3}{2}} \Delta u_{q-\frac{3}{2}}^{n} \\
& \leq 0
\end{aligned}
$$

Similarly, if

$$
\mu=1,\left\{x_{q_{q_{1}}^{\prime}}, \cdots, x_{r_{r_{1}}^{\prime}}\right\}=\left\{x_{r+2}\right\}
$$

then, by the definition of the ridge, we have $u_{r-2}^{n} \leq u_{r-1}^{n} \leq u_{r}^{n} \leq u_{r+1}^{n}<u_{r+2}^{n}$, which derives the following contradiction:

$$
\begin{aligned}
0 & >u_{r+1}^{n+1}-u_{r}^{n+1} \\
& =\left(1-C_{r+\frac{1}{2}}-D_{r+\frac{1}{2}}\right) \Delta u_{r+\frac{1}{2}}^{n}+D_{r+\frac{3}{2}} \Delta u_{r+\frac{3}{2}}^{n}+C_{r-\frac{1}{2}} \Delta u_{r-\frac{1}{2}}^{n} \\
& \geq 0 .
\end{aligned}
$$

Clearly, the ridge satisfies (i) in the Definition 2.2. The ridge also satisfies (ii). In fact, the satisfaction of the Case 1 follows directly from (2.10). In the Case 2, if $\Delta u_{q+\frac{1}{2}}^{n} \geq 0$, then $\Delta u_{q+\frac{1}{2}}^{n} \leq-\Delta u_{q-\frac{1}{2}}^{n}$; otherwise, it derives a contradiction:

$$
\begin{aligned}
0 & <-\left(u_{q+1}^{n+1}-u_{q}^{n+1}\right) \\
& \leq-\left(1-C_{q-\frac{1}{2}}-C_{q+\frac{1}{2}}-D_{q+\frac{1}{2}}\right) \Delta u_{q+\frac{1}{2}}^{n}-D_{q+\frac{3}{2}} \Delta u_{q+\frac{3}{2}}^{n} \\
& \leq 0 .
\end{aligned}
$$


Thus,

$$
V_{\bar{E}}(n+1)-V_{\bar{E}}(n)=u_{q}^{n+1}-u_{q-1}^{n} \leq\left(1-C_{q-\frac{1}{2}}-D_{q+\frac{1}{2}}\right) u_{q-\frac{1}{2}}^{n} \leq 0
$$

as desired. If $\Delta u_{q+\frac{1}{2}}^{n}<0$, we have

$$
V_{\bar{E}}(n+1)-V_{\bar{E}}(n)=u_{q}^{n+1}-u_{q-1}^{n}=\left(1-C_{q-\frac{1}{2}}\right) u_{q-\frac{1}{2}}^{n}+D_{q+\frac{1}{2}} \Delta u_{q+\frac{1}{2}}^{n} \leq 0
$$

as well. Therefore, the Case 2 satisfies (ii) and similarly, we can show the satisfaction (ii) of the Case 3 . For the Case 4, if $P_{\bar{E}}(n)=\left\{x_{q-1}\right\}$, the proof is similar to the Case 2; if $P_{\bar{E}}(n)=\left\{x_{r+1}\right\}$, the proof is similar to the Case 3 .

Step 3. In this step, we verify that the paths constructed in the Step 2 satisfy the property (iii) of the Definition 2.2. Since the Case 1 is irrelevant to this property, we start with the Case 2. We claim that if $u_{q}^{n} \geq u_{q}^{n+1}$, then $c=1$; if $u_{q}^{n}<u_{q}^{n+1}$, then $c=\frac{1}{1-C_{q-\frac{1}{2}}-D_{q+\frac{1}{2}}}$. Indeed, $u_{q}^{n} \geq u_{q}^{n+1}$ implies that

$$
\left|V_{\bar{E}}(n+1)-V_{\bar{E}}(n)\right|=u_{q-1}^{n}-u_{q}^{n+1} \geq u_{q-1}^{n}-u_{q}^{n}=\left|u_{q-1}^{n}-u_{q}^{n}\right| .
$$

Next, $u_{q}^{n}<u_{q}^{n+1}$ implies that if $u_{q+1}^{n} \leq u_{q}^{n}$, then

$$
\begin{aligned}
\left|V_{\bar{E}}(n+1)-V_{\bar{E}}(n)\right| & =u_{q-1}^{n}-u_{q}^{n}+u_{q}^{n}-u_{q}^{n+1} \\
& =-\Delta u_{q-\frac{1}{2}}^{n}+C_{q-\frac{1}{2}} \Delta u_{q-\frac{1}{2}}^{n}-D_{q+\frac{1}{2}} \Delta u_{q+\frac{1}{2}}^{n} \\
& \geq\left(1-C_{q-\frac{1}{2}}\right)\left(-\Delta u_{q-\frac{1}{2}}^{n}\right) \\
& \geq\left(1-C_{q-\frac{1}{2}}-D_{q+\frac{1}{2}}\right)\left|u_{q-1}^{n}-u_{q}^{n}\right| ;
\end{aligned}
$$

if $u_{q+1}^{n}>u_{q}^{n}$, we have $\Delta u_{q+\frac{1}{2}}^{n} \leq-\Delta u_{q-\frac{1}{2}}^{n}$ (otherwise, it yields a contraction (2.17)), and therefore

$$
\begin{aligned}
\left|V_{\bar{E}}(n+1)-V_{\bar{E}}(n)\right| & =-\Delta u_{q-\frac{1}{2}}^{n}+C_{q-\frac{1}{2}} \Delta u_{q-\frac{1}{2}}^{n}-D_{q+\frac{1}{2}} \Delta u_{q+\frac{1}{2}}^{n} \\
& \geq\left(1-C_{q-\frac{1}{2}}-D_{q+\frac{1}{2}}\right)\left|u_{q-1}^{n}-u_{q}^{n}\right| .
\end{aligned}
$$

For the Case 3, we claim that if $u_{q}^{n} \geq u_{q}^{n+1}$, then $c=1$ and if $u_{q}^{n}<u_{q}^{n+1}$, then $c=\frac{1}{1-C_{q-\frac{1}{2}}-D_{q+\frac{1}{2}}}$. Indeed, $u_{q}^{n} \geq u_{q}^{n+1}$ implies that

$$
\left|V_{\bar{E}}(n+1)-V_{\bar{E}}(n)\right|=u_{q+1}^{n}-u_{q}^{n+1} \geq u_{q+1}^{n}-u_{q}^{n}=\left|u_{q+1}^{n}-u_{q}^{n}\right| .
$$

Note that Case 3 implies that $\Delta u_{q+\frac{1}{2}}^{n} \geq 0$. Now $u_{q}^{n}<u_{q}^{n+1}$ ensures that if $\Delta u_{q-\frac{1}{2}}^{n} \geq 0$, then

$$
\begin{aligned}
\left|V_{\bar{E}}(n+1)-V_{\bar{E}}(n)\right| & =u_{q+1}^{n}-u_{q}^{n}+u_{q}^{n}-u_{q}^{n+1} \\
& =C_{q-\frac{1}{2}} \Delta u_{q-\frac{1}{2}}^{n}+\left(1-D_{q+\frac{1}{2}}\right) \Delta u_{q+\frac{1}{2}}^{n} \\
& \geq\left(1-D_{q+\frac{1}{2}}\right) \Delta u_{q+\frac{1}{2}}^{n} \\
& \geq\left(1-C_{q-\frac{1}{2}}-D_{q+\frac{1}{2}}\right)\left|u_{q+1}^{n}-u_{q}^{n}\right| ;
\end{aligned}
$$

if $\Delta u_{q-\frac{1}{2}}^{n}<0$, we have $-\Delta u_{q-\frac{1}{2}}^{n} \leq \Delta u_{q+\frac{1}{2}}^{n}$; otherwise, it derives a contradiction:

$$
\begin{aligned}
0 & <u_{q}^{n+1}-u_{q-1}^{n+1} \\
& =\left(1-C_{q-\frac{1}{2}}-D_{q-\frac{1}{2}}\right) \Delta u_{q-\frac{1}{2}}^{n}+C_{q-\frac{3}{2}} \Delta u_{q-\frac{3}{2}}^{n}+D_{q+\frac{1}{2}} \Delta u_{q+\frac{1}{2}}^{n} \\
& <\left(1-C_{q-\frac{1}{2}}-D_{q-\frac{1}{2}}-D_{q+\frac{1}{2}}\right) \Delta u_{q-\frac{1}{2}}^{n}+D_{q-\frac{3}{2}} \Delta u_{q-\frac{3}{2}}^{n}<0 .
\end{aligned}
$$


Now,

$$
\begin{aligned}
\left|V_{\bar{E}}(n+1)-V_{\bar{E}}(n)\right| & =C_{q-\frac{1}{2}} \Delta u_{q-\frac{1}{2}}^{n}+\left(1-D_{q+\frac{1}{2}}\right) \Delta u_{q+\frac{1}{2}}^{n} \\
& \geq\left(1-C_{q-\frac{1}{2}}-D_{q+\frac{1}{2}}\right)\left|u_{q+1}^{n}-u_{q}^{n}\right| .
\end{aligned}
$$

Finally, we deal with the Case 4 . Without loss the generality, we consider the generic case: $q=r$. Then we have $u_{q}^{n+1} \geq u_{q}^{n}$, which follows easily from (2.10). Thus $c=\frac{1}{1-C_{q-\frac{1}{2}}-D_{q+\frac{1}{2}}}$. The proofs of the instances $u_{q-1}^{n} \geq u_{q+1}^{n}$ and $u_{q-1}^{n} \leq u_{q+1}^{n}$ are similar to that of the Case 2 and the Case 3 respectively for the Case 4.

Step 4. In this step, we verify the property (iv) in the Definition 2.2. Without loss of generality, it suffices to consider the case that $\left\{x_{q}, \cdots, x_{r}\right\}$ is an $\bar{E}$-stencil of $u$ at $t=t_{n+1}$ and $\left\{x_{r+1}, \cdots, x_{r+m}\right\}$ is an $\underline{E}$-stencil of $u$ at $t=t_{n+1}$. Suppose that $\left\{x_{q^{\prime}}, \cdots, x_{r^{\prime}}\right\}$ and $\left\{x_{q^{\prime \prime}}, \cdots, x_{r^{\prime \prime}}\right\}$ are the choices of the $x$-projection at $t=t_{n}$ for the ridge and the trough, respectively. We need to show that $r^{\prime}<q^{\prime \prime}$. In fact, this property follows directly from the construction given in the Step 2 and the implications of the third to the last in the Step 1. The proof is completed. $\square$

In terms of the local CFL numbers, the following result is an easy consequence of the Theorem 2.3.

Corollary 2.4. The sufficient conditions for the schemes (2.7)-(2.8) to be extremum traceable are the following inequalities:

$$
\nu_{k+\frac{1}{2}}^{+}-\nu_{k+\frac{1}{2}}^{-} \leq \frac{2}{2+\Phi}, \quad \text { for all } k,
$$

where $\Phi$ is given by (2.9); when $u_{k}$ is an extremum, there is a constant $\mu, 0 \leq \mu<1$, such that

$$
2\left(\nu_{k \pm \frac{1}{2}}^{+}-\nu_{k \pm \frac{1}{2}}^{-}\right) \leq \frac{\mu}{2}
$$

and

$$
\max \left\{\nu_{k-\frac{1}{2}}^{+}, \nu_{k \pm \frac{3}{2}}^{+},-\nu_{k+\frac{3}{2}}^{-},-\nu_{k+\frac{1}{2}}^{-}\right\} \leq \frac{\mu}{6} .
$$

Proof. Indeed, for all $k$, by Sweby [12], we have $C_{k+\frac{1}{2}} \geq 0, D_{k+\frac{1}{2}} \geq 0$, and

$$
C_{k+\frac{1}{2}}+D_{k+\frac{1}{2}} \leq\left(\nu_{k+\frac{1}{2}}^{+}-\nu_{k+\frac{1}{2}}^{-}\right)\left(\frac{2+\Phi}{2}\right) .
$$

When $u_{k}$ is an extremum, following the equalities:

$$
\begin{array}{ll}
C_{k-\frac{1}{2}}=\nu_{k-\frac{1}{2}}^{+}\left[1-\alpha_{k-\frac{1}{2}}^{+} \varphi\left(r_{k-1}^{+}\right)\right], & D_{k+\frac{1}{2}}=-\nu_{k+\frac{1}{2}}^{-}\left[1-\alpha_{k+\frac{1}{2}}^{-} \varphi\left(r_{k+1}^{-}\right)\right], \\
C_{k+\frac{1}{2}}=\nu_{k+\frac{1}{2}}^{+}\left[1+\alpha_{k+\frac{1}{2}}^{+} \frac{\varphi\left(r_{k+1}^{+}\right)}{r_{k+1}^{+}}\right], & D_{k-\frac{1}{2}}=-\nu_{k-\frac{1}{2}}^{-}\left[1+\alpha_{k-\frac{1}{2}}^{-} \frac{\varphi\left(r_{k-1}^{-}\right)}{r_{k-1}^{-}}\right],
\end{array}
$$

we have arrived

$$
2 C_{k-\frac{1}{2}}+D_{k-\frac{1}{2}} \leq 2\left(\nu_{k-\frac{1}{2}}^{+}-\nu_{k-\frac{1}{2}}^{-}\right) \leq \frac{\mu}{2}<1,
$$




$$
\begin{aligned}
& C_{k+\frac{1}{2}}+2 D_{k+\frac{1}{2}} \leq 2\left(\nu_{k+\frac{1}{2}}^{+}-\nu_{k+\frac{1}{2}}^{-}\right) \leq \frac{\mu}{2}<1, \\
& C_{k+\frac{1}{2}}+D_{k+\frac{1}{2}}+D_{k+\frac{3}{2}}+C_{k-\frac{1}{2}} \\
\leq & 2\left(\nu_{k+\frac{1}{2}}^{+}-\nu_{k+\frac{1}{2}}^{-}\right)+\nu_{k+\frac{1}{2}}^{-}-2 \nu_{k+\frac{3}{2}}^{-}+\nu_{k-\frac{1}{2}}^{+} \leq \mu<1, \\
& C_{k-\frac{1}{2}}+D_{k-\frac{1}{2}}+D_{k+\frac{1}{2}}+C_{k-\frac{3}{2}} \\
\leq & 2\left(\nu_{k-\frac{1}{2}}^{+}-\nu_{k-\frac{1}{2}}^{-}\right)-\nu_{k-\frac{1}{2}}^{+}-\nu_{k+\frac{1}{2}}^{-}+2 \nu_{k-\frac{3}{2}}^{+} \leq \mu<1,
\end{aligned}
$$

and so on, which are desired. $\square$

The next Lemma concerns the flux limiter methods when the building block of the schemes is the Godunov [3] or Engquist-Osher [2] scheme.

The Godunov scheme:

$$
g^{G o d}\left(u_{j}, u_{j+1}\right)=\left\{\begin{array}{lll}
\min _{u_{j} \leq w \leq u_{j+1}} f(w) & \text { when } \quad u_{j} \leq u_{j+1}, \\
\max _{u_{j} \geq w \geq u_{j+1}} f(w) & \text { when } \quad u_{j} \geq u_{j+1}
\end{array}\right.
$$

The Engquist-Osher scheme:

$$
g^{E O}\left(u_{j}, u_{j+1}\right)=\int_{0}^{u_{j}} \max \left(f^{\prime}(w), 0\right) d w+\int_{0}^{u_{j+1}} \min \left(f^{\prime}(w), 0\right) d w+f(0) .
$$

Lemma 2.5. The schemes (2.7)-(2.8) with the building block of Godunov or Engquist-Osher scheme are extremum traceable, provided that

$$
\nu_{k+\frac{1}{2}}^{+}-\nu_{k+\frac{1}{2}}^{-} \leq \frac{2}{2+\Phi} \quad \text { for all } k,
$$

where $\Phi$ is given by (2.9); and when $u_{k}$ is an extremum, there is a constant $\mu, 0 \leq$ $\mu<1$, such that $\lambda K^{\prime}=\lambda \max _{u_{k-2} \leq w \leq u_{k+2}}\left|f^{\prime}(w)\right| \leq \frac{\mu}{6}$.

Proof. By the Corollary 2.4, it suffices to show that when $u_{k}$ is an extremum, we have

$$
\left(\nu_{k \pm \frac{1}{2}}^{+}-\nu_{k \pm \frac{1}{2}}^{-}\right) \leq \lambda K^{\prime}
$$

which can be easily verified. In fact, (2.24) holds for all $k$, when he building block of the schemes (2.7)-(2.8) is Godunov or Engquist-Osher scheme. Thus, the proof is completed.

3. The convergence with van Leer's flux limiter. The following separation property at the spatial extrema asserts that, at the next time level, the values of maximum (minimum) values of the numerical solutions are not increasing (decreasing). Similar conditions can been found in [13], where these types of properties have been used to characterize the convenient TVD conditions by E. Tadmor.

Assumption 3.1. The numerical fluxes $g_{k+\frac{1}{2}}^{n},-\infty<k<\infty$, satisfy

$$
g_{k+\frac{1}{2}}^{n} \geq f\left(u_{k}^{n}\right) \geq g_{k-\frac{1}{2}}^{n} \quad \text { if } \quad u_{k}^{n} \geq u_{k \pm 1}^{n}
$$


and

$$
g_{k+\frac{1}{2}}^{n} \leq f\left(u_{k}^{n}\right) \leq g_{k-\frac{1}{2}}^{n} \quad \text { if } \quad u_{k}^{n} \leq u_{k \pm 1}^{n} .
$$

Lemma 3.2. The scheme (2.7)-(2.8) satisfies the Assumption 3.1.

Proof. If $u_{k} \geq u_{k \pm 1}$, then

$$
\begin{aligned}
g_{k+\frac{1}{2}} & =g_{k+\frac{1}{2}}^{E}-\varphi\left(r_{k+1}^{-}\right) \alpha_{k+\frac{1}{2}}^{-}\left(\Delta f_{k+\frac{1}{2}}\right)^{-} \\
& \geq g_{k+\frac{1}{2}}^{E}-\left(g_{k+\frac{1}{2}}^{E}-f\left(u_{k}\right)\right)=f\left(u_{k}\right),
\end{aligned}
$$

and

$$
\begin{aligned}
g_{k-\frac{1}{2}} & =g_{k-\frac{1}{2}}^{E}+\varphi\left(r_{k-1}^{+}\right) \alpha_{k-\frac{1}{2}}^{+}\left(\Delta f_{k-\frac{1}{2}}\right)^{+} \\
& \leq g_{k-\frac{1}{2}}^{E}+\left(\Delta f_{k-\frac{1}{2}}\right)^{+}=f\left(u_{k}\right) .
\end{aligned}
$$

Similarly, we can show that if $u_{k}^{n} \leq u_{k \pm 1}^{n}$, then $g_{k+\frac{1}{2}}^{n} \leq f\left(u_{k}^{n}\right) \leq g_{k-\frac{1}{2}}^{n}$.

Denote $\tilde{v}_{j}=H\left(v_{j-p}, \cdots, v_{j+p} ; \lambda\right)$ (see (1.3)), $\bar{v}_{j}=\frac{v_{j}+\tilde{v}_{j}}{2}$ for any collection of data $\left\{v_{j}\right\}$, and $f[w ; L, R]$ be the linear function interpolating $f(w)$ at $w=L$ and $w=R$. In this section, we assume that $f^{\prime \prime}(w) \geq 0$.

Definition 3.3 (see Definition $2.20[16]$ ). We call an ordered pair of numbers $\{L, R\}$ a rarefying pair if $L<R$ and $f[w ; L, R]>f(w)$ when $L<w<R$. We call a collection of data $\Gamma=\left\{v_{j}\right\}_{j=I-p}^{J+p}$ an $\varepsilon$-rarefying collection of the scheme to the rarefying pair $\{L, R\}$ if, for $\varepsilon>0$,

(i) $L=v_{I} \leq v_{I+1} \leq \cdots \leq v_{J}=R$;

(ii) $\tilde{v}_{I} \leq \tilde{v}_{I+1} \leq \cdots \leq \tilde{v}_{J},\left|L-\tilde{v}_{I}\right|<\varepsilon,\left|R-\tilde{v}_{J}\right|<\varepsilon$;

(iii) either $v_{I-1} \geq v_{I}$ or $v_{I}=v_{I+1}$; and either $v_{J+1} \leq v_{J}$ or $v_{J-1}=v_{J}$.

The conditions (i) and (ii) imply that $\bar{v}_{I} \leq \bar{v}_{I+1} \leq \cdots \leq \bar{v}_{J},\left|L-\bar{v}_{I}\right|<\frac{\varepsilon}{2}$, and $\mid R-$ $\bar{v}_{J} \mid<\frac{\varepsilon}{2}$. We define the piecewise constant function $g_{\Gamma}$ associated with the $\varepsilon$-rarefying collection $\Gamma$ as follows:

$$
g_{\Gamma}(w)=g_{j+\frac{1}{2}}[v] \quad \text { for } w \in\left(\bar{v}_{j}, \bar{v}_{j+1}\right), \quad I \leq j \leq J-1 .
$$

A 0-rarefying collection $\Gamma=\left\{v_{j}\right\}_{j=I-2}^{J+2}$ of the scheme to the pair $\{L, R\}$ that satisfies

$$
L=v_{I-2}=v_{I-1}=v_{I}=v_{I+1} \leq \cdots \leq v_{J-1}=v_{J}=v_{J+1}=v_{J+2}=R
$$

is called a normal collection.

Theorem 3.4 (see Theorem 2.21[16]). An extremum traceable scheme that satisfies Assumption 3.1 converges for convex conservation laws if, for every rarefying pair $\{L, R\}$ and $\varepsilon$-rarefying collection to the pair,

$$
\int_{L}^{R} f[w ; L, R] d w-\int_{\bar{v}_{I}}^{\bar{v}_{J}} g_{\Gamma}(w) d w>\delta
$$

for some constant $\delta>0$ depending only on the exact flux $f$, the numerical flux function $g$, and the two numbers $L$ and $R$, provided that $\varepsilon$ is sufficiently small. 
For the class of flux limiter schemes concerned, this convergence criterion can be simplified by the following Lemma.

LEMMA 3.5. An extremum traceable scheme of the form (2.7)-(2.8) converges for convex conservation laws, provided that for each rarefying pair $\{L, R\}$ there is a constant $\delta>0$ such that the inequality (3.2) holds for all normal corrections of the scheme to the pair $\{L, R\}$.

Proof. Let $\Lambda=\left\{\kappa_{P-2}, \cdots, \kappa_{Q+2}\right\}$ be an arbitrary $\varepsilon$-rarefying collection of the scheme to the pair $\{L, R\}$. Let

$$
S^{\prime}=\int_{\bar{\kappa}_{P}}^{\bar{\kappa}_{Q}} g_{\Lambda}(w) d w=\sum_{j=P}^{Q-1}\left(\bar{\kappa}_{j+1}-\bar{\kappa}_{j}\right) g_{j+\frac{1}{2}}[\kappa] .
$$

By (i) and (iii) of Definition 3.3, either $\kappa_{P}$ or $\kappa_{P+1}$ is a minimum. In either case, Assumption 3.1 and the condition (ii) of Definition 3.3 imply that

$$
\varepsilon>\left|L-\tilde{\kappa}_{P}\right|=\left|\tilde{\kappa}_{P}-\kappa_{P}\right|=\lambda\left|g_{P+\frac{1}{2}}[\kappa]-g_{P-\frac{1}{2}}[\kappa]\right| \geq \lambda\left|g_{P \pm \frac{1}{2}}[\kappa]-f(L)\right| .
$$

Similarly, we have

$$
\varepsilon>\left|R-\tilde{\kappa}_{Q}\right| \geq \lambda\left|g_{Q \pm \frac{1}{2}}[\kappa]-f(R)\right|
$$

Next, we construct a normal collection $\Gamma=\left\{v_{j}\right\}_{j=I-2}^{J+2}$ as follows. First, let $I=P-1$ and $J=Q+1$ and we also set $v_{I-2}=v_{I-1}=v_{I}=L, v_{J}=v_{J+1}=v_{J+2}=R$, and $v_{j}=\kappa_{j}$ for $I+1 \leq j \leq J-1$. Then, we have

$$
g_{I \pm \frac{1}{2}}[v]=f(L) \quad \text { and } \quad g_{J \pm \frac{1}{2}}[v]=f(R)
$$

which imply that,

$$
\bar{v}_{I}=\tilde{v}_{I}=v_{I}=L \quad \text { and } \quad \bar{v}_{J}=\tilde{v}_{J}=v_{J}=R .
$$

Thus, $\Gamma=\left\{v_{j}\right\}_{j=I-2}^{J+2}$ indeed is a normal collection. Secondly, let $G$ be the Lipschitz constant of the numerical flux $g$, and $K=\max \{|f(L)|,|f(R)|\}+G(R-L)$. Denote

$$
S=\int_{L}^{R} g_{\Gamma}(w) d w=\sum_{j=I}^{J-1}\left(\bar{v}_{j+1}-\bar{v}_{j}\right) g_{j+\frac{1}{2}}[v],
$$

then a-priori estimate $\left|S-S^{\prime}\right| \leq 3 K \varepsilon$ holds. Let $\delta^{\prime}$ be a constant such that for all normal collections of the scheme to the pair $\{L, R\}$ the inequality (3.2) holds for $\delta=\delta^{\prime}$. Thus, for $\delta=\delta^{\prime}$, the inequality (3.2) also holds for the normal collection $\Gamma=\left\{v_{j}\right\}_{j=I-2}^{J+2}$. Therefore, for $\delta=\frac{\delta^{\prime}}{2}$, the inequality (3.2) holds for all $\varepsilon$-collection of the scheme to the pair $\{L, R\}$ provided that $\varepsilon \leq \frac{\delta}{3 K}$. It remains to show the a-priori estimate. Notice that $g_{j+\frac{1}{2}}[\kappa]=g_{j+\frac{1}{2}}[v]$, for $P \leq j \leq Q-1$, and therefore, $\bar{\kappa}_{j}$ for $P+1 \leq j \leq Q-1$ are independent of $\kappa_{i}$ for $i<P$ or $i>Q$. Thus, $\bar{\kappa}_{j}=\bar{v}_{j}$ for $P+1 \leq j \leq Q-1$, and we have

$$
\begin{aligned}
\left|S-S^{\prime}\right| & \leq\left|\bar{v}_{I+1}-\bar{v}_{I}\right|\left|g_{I+\frac{1}{2}}[v]\right|+\left|\bar{v}_{I+1}-\bar{\kappa}_{I+1}\right|\left|g_{I+\frac{3}{2}}[v]\right| \\
& +\left|\bar{v}_{J}-\bar{v}_{J-1}\right|\left|g_{J-\frac{1}{2}}[v]\right|+\left|\bar{v}_{J-1}-\bar{\kappa}_{J-1}\right|\left|g_{J-\frac{3}{2}}[v]\right| .
\end{aligned}
$$


The relationship of $\Lambda$ and $\Gamma$ and the inequalities (3.4)-(3.7) yield:

$$
\begin{gathered}
\left|\bar{v}_{I+1}-\bar{\kappa}_{I+1}\right|<\frac{\varepsilon}{2}, \quad\left|\bar{v}_{J-1}-\bar{\kappa}_{J-1}\right|<\frac{\varepsilon}{2}, \\
\left|\bar{v}_{I+1}-\bar{v}_{I}\right|=\left|\bar{v}_{I+1}-L\right| \leq\left|\bar{v}_{I+1}-\bar{\kappa}_{I+1}\right|+\left|\bar{\kappa}_{I+1}-L\right|<\varepsilon,
\end{gathered}
$$

and

$$
\left|\bar{v}_{J}-\bar{v}_{J-1}\right|=\left|\bar{v}_{J-1}-R\right| \leq\left|\bar{v}_{J-1}-\bar{\kappa}_{J-1}\right|+\left|\bar{\kappa}_{Q}-R\right|<\varepsilon .
$$

Finally, $\left|S-S^{\prime}\right|<3 K \varepsilon$ follows from the inequalities (3.9)-(3.12).

For a normal collection $\Gamma=\left\{v_{j}\right\}_{j=I-2}^{J+2}$, we denote the vertex $\left(v_{j}, f\left(v_{j}\right)\right)$ by $V_{j}$ and the area of convex polygon $V_{j_{1}} V_{j_{2}} \cdots V_{j_{r}}$ by $S_{j_{1}, \ldots, j_{r}}$. Let $\sigma_{\Gamma}=\max _{I-2 \leq j \leq J+2}\left|\nu_{j \pm \frac{1}{2}}^{ \pm}\right|$, and let

$$
\alpha_{j}= \begin{cases}0.5 & \text { if } \Delta v_{j-2}=\Delta v_{j+1}=0 \\ 1 & \text { otherwise }\end{cases}
$$

When the building block of the schemes (2.7)-(2.8) is the Godunov (2.21) or Engquist-Osher (2.22) and $\varphi$ is van Leer's flux (3.14), we have the following very important estimate. The proof will be given at the end of this section.

Lemma 3.6. Let $\Gamma=\left\{v_{j}\right\}_{j=I-2}^{J+2}$ be a normal collection to a rarefying pair $\{L, R\}$. Then the numerical solutions of the schemes (2.7)-(2.8) for convex conservation laws satisfy, for a sufficiently small $\sigma_{\Gamma}$, the following inequality

$$
\int_{L}^{R}\left(f[w ; L, R]-g_{\Gamma}\right) d w \geq S_{I, I+1, \ldots, J}-\sum_{j=I+1}^{J-1} \alpha_{j} S_{j-1, j, j+1} .
$$

Lemma 3.7 ( see Lemma $3.7[16]$ ). We have

$$
S_{I, I+1, \ldots, J}-\sum_{j=I+1}^{J-1} S_{j-1, j, j+1} \geq S_{I, i, i+1, J}-\left(S_{I, i, i+1}+S_{i, i+1, J}\right)
$$

for $I<i<J-1$.

Let $\sigma=\lambda \max _{w}\left|f^{\prime}(w)\right|$. For van Leer's flux limiter [12]:

$$
\varphi_{V L}(r)= \begin{cases}0 & r \leq 0 \\ \frac{2 r}{1+r} & r>0\end{cases}
$$

we have obtained the following entropy convergence result. The proof is similar to the one given by Yang [16] for the MUSCL schemes.

THEOREM 3.8. The numerical solutions of the schemes (2.7)-(2.8), for the convex problems (1.1), converge provided that $\varphi=\varphi_{V L}$ is van Leer's flux limiter given by (3.14), $g^{E}(\cdot, \cdot)$ is either the numerical flux of the Godunov scheme given by (2.21) or Engquist-Osher scheme given by (2.22), and $\sigma$ is sufficiently small.

Proof. For each normal collection $\Gamma=\left\{v_{i}\right\}_{i=I-2}^{J+2}$ to a rarefying pair $\{L, R\}$, we set

$$
d_{1}(\Gamma)=\max _{I \leq i \leq J} \min \left(v_{i}-L, R-v_{i}\right) .
$$


Since $J-I$ is finite, $d_{1}(\Gamma)=\min \left(v_{j}-L, R-v_{j}\right)$ for some $j$ between $I$ and $J$. We then let

$$
d_{2}(\Gamma)=\max _{I \leq i \leq J, i \neq j} \min \left(v_{i}-L, R-v_{i}\right) .
$$

We also have $d_{2}(\Gamma)=\min \left(v_{k}-L, R-v_{k}\right)$ for some $k \neq j$ between $I$ and $J$. Clearly, we can choose $j$ and $k$ so that $|j-k|=1$.

To complete the proof, we argue by contradiction. Hence, we assume that for certain convex $f$, the scheme of the form (2.7)-(2.8) does not converge. By Lemma 3.5 , there is a rarefying pair $\{L, R\}$ such that for each $\delta>0$, there is a normal collection $\Gamma=\left\{v_{j}\right\}_{j=I-2}^{J+2}$ of the scheme to the pair that satisfies

$$
\int_{L}^{R}\left\{f[w ; L, R]-g_{\Gamma}(w)\right\} d w \leq \delta .
$$

It follows that there is a sequence of normal collections $\left\{\Gamma_{\nu}\right\}_{\nu=1}^{\infty}$, where $\Gamma_{\nu}=$ $\left\{v_{j}^{\nu}\right\}_{j=I^{\nu}-2}^{J^{\nu}+2}$ such that

$$
\lim _{\nu \rightarrow \infty} \int_{L}^{R}\left\{f[w ; L, R]-g_{\Gamma_{\nu}}(w)\right\} \leq 0 .
$$

The following three cases exhaust all possibilities.

Case 1. $\lim \sup _{\nu \rightarrow \infty} d_{2}\left(\Gamma_{\nu}\right)>0$. Set $\rho=\frac{1}{2} \lim \sup _{\nu \rightarrow \infty} d_{2}\left(\Gamma_{\nu}\right)$. Then, there is a subsequence of the normal collections, still denoted by $\left\{\Gamma_{\nu}\right\}_{\nu=1}^{\infty}$, and a corresponding sequence of integers $\{i(\nu)\}_{\nu=1}^{\infty}$ such that

$$
L+\rho \leq v_{i(\nu)}^{\nu} \leq v_{i(\nu)+1}^{\nu} \leq R-\rho,
$$

and $\sup _{\nu} \sigma_{\Gamma_{\nu}} \leq \sigma$. For simplicity, we fix a $\nu$ and drop it from the notation. Set $\gamma=f\left[\frac{L+R}{2} ; L, R\right]-f\left(\frac{L+R}{2}\right)$. It is a positive constant since $\{L, R\}$ is a rarefying pair. Applying Lemmas 3.6 and 3.7, we have

$$
\begin{aligned}
& \int_{L}^{R}\left\{f[w ; L, R]-g_{\Gamma_{\nu}}(w)\right\} d w \geq S_{I, i, i+1, J}-\left(S_{I, i, i+1}+S_{i, i+1, J}\right) \\
= & \frac{1}{2}\left\{\left(v_{i}-v_{I}\right)\left(f\left[v_{i+1} ; L, R\right]-f\left(v_{i+1}\right)\right)+\left(v_{J}-v_{i+1}\right)\left(f\left[v_{i} ; L, R\right]-f\left(v_{i}\right)\right)\right\} \\
> & \eta
\end{aligned}
$$

if $\eta=2 \rho^{2} \gamma /(R-L)$. This contradicts (3.15).

Case 2. $\quad \lim \sup _{\nu \rightarrow \infty} d_{1}\left(\Gamma_{\nu}\right)>\limsup _{\nu \rightarrow \infty} d_{2}\left(\Gamma_{\nu}\right)=0$. Set $\rho=$ $\frac{1}{2} \lim \sup _{\nu \rightarrow \infty} d_{1}\left(\Gamma_{\nu}\right)$. Then, there is a subsequence of the normal collections, still denoted by $\left\{\Gamma_{\nu}\right\}_{\nu=1}^{\infty}$, and a corresponding sequence of integers $\left\{i^{\nu}\right\}_{\nu=1}^{\infty}$ such that $\lim _{\nu \rightarrow \infty} v_{i^{\nu}-1}^{\nu}=L, \lim _{\nu \rightarrow \infty} v_{i^{\nu}+1}^{\nu}=R$, and $\lim _{\nu \rightarrow \infty} v_{i^{\nu}}^{\nu}=v \in[L+\rho, R-\rho]$. We then have

$$
\int_{L}^{R}\left(f[w ; L, R]-g_{\Gamma_{\nu}}(w)\right) d w \rightarrow \int_{L}^{R}\left(f[w ; L, R]-g_{\Gamma}(w)\right) d w,
$$

where $\Gamma$ is the following normal collection: $I=0, J=4, v_{-2}=v_{-1}=v_{0}=v_{1}=$ $L, v_{2}=v$, and $v_{3}=v_{4}=v_{5}=v_{6}=R$. By Lemma 3.6, we have

$$
\int_{L}^{R}\left(f[w ; L, R]-g_{\Gamma}(w)\right) d w \geq S_{1,2,3}-\alpha_{2} S_{1,2,3}=\frac{1}{2} S_{1,2,3}>0
$$


for $\alpha_{2}=\frac{1}{2}$ since $\Delta v_{0}=\Delta v_{3}=0$. This contradicts (3.15).

Case 3. $\lim \sup _{\nu \rightarrow \infty} d_{1}\left(\Gamma_{\nu}\right)=0$. Then, there exists a sequence of integers $\left\{i^{\nu}\right\}$ with $I^{\nu}+1 \leq i^{\nu}<J^{\nu}-1$ such that $\lim _{\nu \rightarrow \infty} v_{i^{\nu}}^{\nu}=L, \lim _{\nu \rightarrow \infty} v_{i^{\nu}+1}^{\nu}=R$. We then have

$$
\int_{L}^{R}\left(f[w ; L, R]-g_{\Gamma_{\nu}}(w)\right) d w \rightarrow \int_{L}^{R}\left(f[w ; L, R]-g_{\Gamma}(w)\right) d w
$$

where $\Gamma$ is the following normal collection: $I=0, J=3, v_{-2}=v_{-1}=v_{0}=v_{1}=$ $L, v_{2}=v_{3}=v_{4}=v_{5}=R$. In this case, the numerical flux $g_{\Gamma}(w)$ becomes $E$-flux $g^{E}(L, R)$. Hence, we have

$$
\int_{L}^{R}\left(f[w ; L, R]-g_{\Gamma}(w)\right) d w \geq \int_{L}^{R}(f[w ; L, R]-f(w)) d w .
$$

The right-hand side of the inequality is a positive constant since $\{L, R\}$ is a rarefying pair. This contradicts (3.15) again. We have thus completed the proof of Theorem 3.8.

Finally, we finish this section by presenting the proof of Lemma 3.6.

Proof of Lemma 3.6. In the proof, the following inequality is helpful:

$$
\frac{\alpha \beta}{\alpha+\beta} \leq \frac{\alpha+\beta}{4} \quad \forall \alpha, \beta \in \mathbb{R} \quad \text { with } \quad \alpha+\beta>0
$$

Here, we keep the same notations $\left(\Delta f_{j+\frac{1}{2}}\right)^{ \pm}$and $r_{j}^{ \pm}$for $\left\{v_{j}\right\}$ instead of $\left\{u_{j}\right\}$. We also use the following notation for the divided difference:

$$
f_{j+\frac{1}{2}}^{\prime}:=\frac{f\left(v_{j+1}\right)-f\left(v_{j}\right)}{v_{j+1}-v_{j}} .
$$

To justify the inequality (3.13), it suffices to show the following inequality:

$$
\int_{L}^{R} g_{\Gamma}(w) d w-\sum_{j=I}^{J-1} \int_{v_{j}}^{v_{j+1}} f\left[w ; v_{j}, v_{j+1}\right] d w \leq \sum_{j=I+1}^{J-1} \alpha_{j} S_{j-1, j, j+1} .
$$

Without loss of generality, Let $v_{s}$ be a sonic point $\left(f^{\prime}\left(v_{s}\right)=0\right)$ such that $v_{k} \leq$ $v_{s} \leq v_{k+1}$ for some integer $k$ with $I \leq k \leq J-1$. Then for $g^{E}(\cdot, \cdot)$ given by $(2.21)$ or (2.22), we have

$$
\begin{gathered}
\left(\Delta f_{j+\frac{1}{2}}\right)^{+}=0, \quad \text { for } I \leq j \leq k-1 \\
\left(\Delta f_{j+\frac{1}{2}}\right)^{+}=f_{j+\frac{1}{2}}^{\prime} \Delta v_{j+\frac{1}{2}}, \quad \text { for } J-1 \geq j \geq k+1 \\
\left(\Delta f_{j+\frac{1}{2}}\right)^{-}=0, \quad \text { for } J-1 \geq j \geq k+1
\end{gathered}
$$

and

$$
\left(\Delta f_{j+\frac{1}{2}}\right)^{-}=f_{j+\frac{1}{2}}^{\prime} \Delta v_{j+\frac{1}{2}}, \quad \text { for } I \leq j \leq k-1 .
$$


To easy the notations, we denote $f_{j}:=f\left(v_{j}\right), f_{s}:=f\left(v_{s}\right), v_{j \pm \frac{1}{2}}:=\Delta v_{j \pm \frac{1}{2}}=$ $\pm\left(v_{j+1}-v_{j}\right), v_{s+\frac{1}{2}}:=v_{k+1}-v_{s}, v_{s-\frac{1}{2}}:=v_{s}-v_{k}, f_{s+\frac{1}{2}}^{\prime}:=\left(f_{k+1}-f_{s}\right) / v_{s+\frac{1}{2}}, f_{s-\frac{1}{2}}^{\prime}:=$ $\left(f_{s}-f_{k}\right) / v_{s-\frac{1}{2}}, \bar{v}_{j \pm \frac{1}{2}}:=\Delta \bar{v}_{j \pm \frac{1}{2}}, \tilde{v}_{j \pm \frac{1}{2}}:=\Delta \tilde{v}_{j \pm \frac{1}{2}}$, and $f_{j \pm \frac{1}{2}}^{ \pm}:=\left(\Delta f_{j \pm \frac{1}{2}}\right)^{ \pm}$. Then we have

$$
\begin{aligned}
& \sum_{j=I}^{J-1} g_{j+\frac{1}{2}} \bar{v}_{j+\frac{1}{2}}-\sum_{j=I}^{J-1} \int_{v_{j}}^{v_{j+1}} f\left[w ; v_{j}, v_{j+1}\right] d w \\
= & \sum_{j=I}^{J-1} g_{j+\frac{1}{2}} \frac{v_{j+\frac{1}{2}}+\tilde{v}_{j+\frac{1}{2}}}{2}-\sum_{j=I}^{J-1} \frac{f_{j}+f_{j+1}}{2} v_{j+\frac{1}{2}} \\
= & \frac{1}{2}\left(P_{(j \leq k-2)}+P_{k-1}+P_{k}+P_{k+1}+P_{(j \geq k+2)}\right),
\end{aligned}
$$

and the definitions of $P_{(j \leq k-2)}, P_{k-1}, P_{k}, P_{k+1}$ and $P_{(j \geq k+2)}$ will be given shortly.

Recall,

$$
g_{j+\frac{1}{2}}=g_{j+\frac{1}{2}}^{E}+\alpha_{j+\frac{1}{2}}^{+} \varphi\left(r_{j}^{+}\right) f_{j+\frac{1}{2}}^{+}-\alpha_{j+\frac{1}{2}}^{-} \varphi\left(r_{j+1}^{-}\right) f_{j+\frac{1}{2}}^{-},
$$

and by (3.17), we have

$$
\alpha_{j+\frac{1}{2}}^{+} \varphi\left(r_{j}^{+}\right) f_{j+\frac{1}{2}}^{+}=2 \frac{\alpha_{j-\frac{1}{2}}^{+} f_{j-\frac{1}{2}}^{+} \alpha_{j+\frac{1}{2}}^{+} f_{j+\frac{1}{2}}^{+}}{\alpha_{j-\frac{1}{2}}^{+} f_{j-\frac{1}{2}}^{+}+\alpha_{j+\frac{1}{2}}^{+} f_{j+\frac{1}{2}}^{+}} \leq \frac{f_{j-\frac{1}{2}}^{+}+f_{j+\frac{1}{2}}^{+}}{4},
$$

and

$$
-\alpha_{j+\frac{1}{2}}^{-} \varphi\left(r_{j+1}^{-}\right) f_{j+\frac{1}{2}}^{-}=-2 \frac{\alpha_{j+\frac{1}{2}}^{-} f_{j+\frac{1}{2}}^{-} \alpha_{j+\frac{3}{2}}^{-} f_{j+\frac{3}{2}}^{-}}{\alpha_{j+\frac{1}{2}}^{-} f_{j+\frac{1}{2}}^{-}+\alpha_{j+\frac{3}{2}}^{-} f_{j+\frac{3}{2}}^{-}} \leq \frac{-f_{j+\frac{1}{2}}^{-}-f_{j+\frac{3}{2}}^{-}}{4}
$$

also recall that

$$
\tilde{v}_{j+\frac{1}{2}}=v_{j+\frac{1}{2}}-\left(C_{j+\frac{1}{2}}+D_{j+\frac{1}{2}}\right) v_{j+\frac{1}{2}}+D_{j+\frac{3}{2}} v_{j+\frac{3}{2}}+C_{j-\frac{1}{2}} v_{j-\frac{1}{2}} \geq 0 .
$$

With these inequalities and the convexity of the flux, we have derived the following 
estimates.

$$
\begin{aligned}
& P_{(j \leq k-2)}:=\sum_{j=I}^{k-2}\left\{g_{j+\frac{1}{2}}\left[v_{j+\frac{1}{2}}+\tilde{v}_{j+\frac{1}{2}}\right]-\left(f_{j}+f_{j+1}\right) v_{j+\frac{1}{2}}\right\} \\
& =\sum_{j=I}^{k-2}\left\{[ g _ { j + \frac { 1 } { 2 } } ^ { E } + \alpha _ { j + \frac { 1 } { 2 } } ^ { + } \varphi ( r _ { j } ^ { + } ) f _ { j + \frac { 1 } { 2 } } ^ { + } - \alpha _ { j + \frac { 1 } { 2 } } ^ { - } \varphi ( r _ { j + 1 } ^ { - } ) f _ { j + \frac { 1 } { 2 } } ^ { - } ] \left[2 v_{j+\frac{1}{2}}\right.\right. \\
& \left.\left.-\left(C_{j+\frac{1}{2}}+D_{j+\frac{1}{2}}\right) v_{j+\frac{1}{2}}+D_{j+\frac{3}{2}} v_{j+\frac{3}{2}}+C_{j-\frac{1}{2}} v_{j-\frac{1}{2}}\right]-\left(f_{j}+f_{j+1}\right) v_{j+\frac{1}{2}}\right\} \\
& =\sum_{j=I}^{k-2}\left\{\left[g_{j+\frac{1}{2}}^{E}-\alpha_{j+\frac{1}{2}}^{-} \varphi\left(r_{j+1}^{-}\right) f_{j+\frac{1}{2}}^{-}\right]\left[2 v_{j+\frac{1}{2}}-D_{j+\frac{1}{2}} v_{j+\frac{1}{2}}+D_{j+\frac{3}{2}} v_{j+\frac{3}{2}}\right]\right. \\
& \left.-\left(f_{j}+f_{j+1}\right) v_{j+\frac{1}{2}}\right\} \\
& \leq \sum_{j=I}^{k-2}\left\{\left[g_{j+\frac{1}{2}}^{E}+\frac{-f_{j+\frac{1}{2}}^{-}-f_{j+\frac{3}{2}}^{-}}{4}\right]\left[2 v_{j+\frac{1}{2}}-D_{j+\frac{1}{2}} v_{j+\frac{1}{2}}+D_{j+\frac{3}{2}} v_{j+\frac{3}{2}}\right]\right. \\
& \left.-\left(f_{j}+f_{j+1}\right) v_{j+\frac{1}{2}}\right\} \\
& =\frac{1}{4}\left\{\sum _ { j = I } ^ { k - 2 } \left[\left(2 f_{j+\frac{1}{2}}^{\prime} v_{j+\frac{1}{2}}^{2}-2 f_{j+\frac{3}{2}}^{\prime} v_{j+\frac{1}{2}} v_{j+\frac{3}{2}}\right)+\left(-4 f_{j+\frac{1}{2}}^{\prime} v_{j+\frac{1}{2}}^{2}-f_{j-\frac{1}{2}}^{\prime} v_{j-\frac{1}{2}} v_{j+\frac{1}{2}}\right.\right.\right. \\
& \left.\left.\left.+f_{j+\frac{3}{2}}^{\prime} v_{j+\frac{1}{2}} v_{j+\frac{3}{2}}\right) D_{j+\frac{1}{2}}+\left(4 f_{k-1}+f_{k-2}-f_{k}\right) v_{k-\frac{1}{2}} D_{k-\frac{1}{2}}\right]\right\} \\
& \leq \frac{1}{4}\left\{\sum _ { j = I } ^ { k - 2 } \left[f_{j+\frac{3}{2}}^{\prime}\left(v_{j+\frac{1}{2}}-v_{j+\frac{3}{2}}\right)^{2}-f_{k-\frac{1}{2}}^{\prime} v_{k-\frac{1}{2}}^{2}+\left(4 f_{k-1}+f_{k-2}-f_{k}\right) v_{k-\frac{1}{2}} D_{k-\frac{1}{2}}\right.\right. \\
& \left.\left.+\left(-4 f_{j+\frac{1}{2}}^{\prime} v_{j+\frac{1}{2}}^{2}-f_{j-\frac{1}{2}}^{\prime} v_{j-\frac{1}{2}} v_{j+\frac{1}{2}}+f_{j+\frac{3}{2}}^{\prime} v_{j+\frac{1}{2}} v_{j+\frac{3}{2}}\right) D_{j+\frac{1}{2}}\right]\right\} \\
& \leq \frac{1}{4}\left\{\sum_{j=I}^{k-2}\left[\left(-4 f_{j+\frac{1}{2}}^{\prime} v_{j+\frac{1}{2}}^{2}-f_{j-\frac{1}{2}}^{\prime} v_{j-\frac{1}{2}} v_{j+\frac{1}{2}}\right) D_{j+\frac{1}{2}}+\text { k-term of } P_{(j \leq k-2)}\right]\right\},
\end{aligned}
$$

where, k-term of $P_{(j \leq k-2)}:=-f_{k-\frac{1}{2}}^{\prime} v_{k-\frac{1}{2}}^{2}+\left(4 f_{k-1}+f_{k-2}-f_{k}\right) v_{k-\frac{1}{2}} D_{k-\frac{1}{2}}$.

Similarly, we obtain

$$
\begin{aligned}
& P_{(j \geq k+2)}:=\sum_{j=k+2}^{J-1}\left\{g_{j+\frac{1}{2}}\left[v_{j+\frac{1}{2}}+\tilde{v}_{j+\frac{1}{2}}\right]-\left(f_{j}+f_{j+1}\right) v_{j+\frac{1}{2}}\right\} \\
= & \sum_{j=k+2}^{J-1}\left\{\left[g_{j+\frac{1}{2}}^{E}+\alpha_{j+\frac{1}{2}}^{+} \varphi\left(r_{j}^{+}\right) f_{j+\frac{1}{2}}^{+}\right]\left[2 v_{j+\frac{1}{2}}-C_{j+\frac{1}{2}} v_{j+\frac{1}{2}}+C_{j-\frac{1}{2}} v_{j-\frac{1}{2}}\right]\right. \\
& \left.-\left(f_{j}+f_{j+1}\right) v_{j+\frac{1}{2}}\right\} \\
\leq & \frac{1}{4}\left\{\sum_{j=k+2}^{J-1}\left[\left(f_{j+\frac{3}{2}}^{\prime} v_{j+\frac{1}{2}} v_{j+\frac{3}{2}}+4 f_{j+\frac{1}{2}}^{\prime} v_{j+\frac{1}{2}}^{2}\right) C_{j+\frac{1}{2}}+\mathrm{k} \text {-term of } P_{(j \geq k+2)}\right]\right\}
\end{aligned}
$$

where, k-term of $P_{(j \geq k+2)}:=f_{k+\frac{3}{2}}^{\prime} v_{k+\frac{3}{2}}^{2}+\left(4 f_{k+2}-f_{k+1}+f_{k+3}\right) v_{k+\frac{3}{2}} C_{k+\frac{3}{2}}$. 
Now,

$$
\begin{aligned}
P_{k-1}:= & {\left[g_{k-\frac{1}{2}}^{E}+\alpha_{k-\frac{1}{2}}^{+} \varphi\left(r_{k-1}^{+}\right) f_{k-\frac{1}{2}}^{+}-\alpha_{k-\frac{1}{2}}^{-} \varphi\left(r_{k}^{-}\right) f_{k-\frac{1}{2}}^{-}\right]\left[2 v_{k-\frac{1}{2}}\right.} \\
& \left.-\left(C_{k-\frac{1}{2}}+D_{k-\frac{1}{2}}\right) v_{k-\frac{1}{2}}+D_{k+\frac{1}{2}} v_{k+\frac{1}{2}}+C_{k-\frac{3}{2}} v_{k-\frac{3}{2}}\right]-\left(f_{k-1}+f_{k}\right) v_{k-\frac{1}{2}} \\
\leq & {\left[g_{k-\frac{1}{2}}^{E}+\frac{\left.-f_{k-\frac{1}{2}}^{-}-f_{k+\frac{1}{2}}^{-}\right]\left[2 v_{k-\frac{1}{2}}-D_{k-\frac{1}{2}} v_{k-\frac{1}{2}}+D_{k+\frac{1}{2}} v_{k+\frac{1}{2}}\right]-\left(f_{k-1}+f_{k}\right) v_{k-\frac{1}{2}}}{4}=\frac{1}{4}\left[\left(4 f_{k}+f_{k-1}-f_{s}\right)\left(2 v_{k-\frac{1}{2}}-D_{k-\frac{1}{2}} v_{k-\frac{1}{2}}+D_{k+\frac{1}{2}} v_{k+\frac{1}{2}}\right)-4\left(f_{k-1}+f_{k}\right) v_{k-\frac{1}{2}}\right],\right.} \\
P_{k}:= & {\left[g_{k+\frac{1}{2}}^{E}+\alpha_{k+\frac{1}{2}}^{+} \varphi\left(r_{k}^{+}\right) f_{k+\frac{1}{2}}^{+}-\alpha_{k+\frac{1}{2}}^{-} \varphi\left(r_{k+1}^{-}\right) f_{k-\frac{1}{2}}^{-}\right]\left[2 v_{k+\frac{1}{2}}\right.} \\
& \left.\quad-\left(C_{k+\frac{1}{2}}+D_{k+\frac{1}{2}}\right) v_{k+\frac{1}{2}}+D_{k+\frac{3}{2}} v_{k+\frac{3}{2}}+C_{k-\frac{1}{2}} v_{k-\frac{1}{2}}\right]-\left(f_{k}+f_{k+1}\right) v_{k+\frac{1}{2}} \\
= & g_{k+\frac{1}{2}}^{E}\left[2 v_{k+\frac{1}{2}}-C_{k+\frac{1}{2}} v_{k+\frac{1}{2}}-D_{k+\frac{1}{2}} v_{k+\frac{1}{2}}\right]-\left(f_{k}+f_{k+1}\right) v_{k-\frac{1}{2}} \\
= & \frac{1}{4}\left[4 f_{s}\left(2 v_{k+\frac{1}{2}}-C_{k+\frac{1}{2}} v_{k+\frac{1}{2}}-D_{k+\frac{1}{2}} v_{k+\frac{1}{2}}\right)-4\left(f_{k}+f_{k+1}\right) v_{k+\frac{1}{2}}\right],
\end{aligned}
$$

and

$$
\begin{aligned}
P_{k+1}:= & {\left[g_{k+\frac{3}{2}}^{E}+\alpha_{k+\frac{3}{2}}^{+} \varphi\left(r_{k+1}^{+}\right) f_{k+\frac{3}{2}}^{+}-\alpha_{k+\frac{3}{2}}^{-} \varphi\left(r_{k+2}^{-}\right) f_{k+\frac{3}{2}}^{-}\right]\left[2 v_{k+\frac{3}{2}}\right.} \\
& \left.-\left(C_{k+\frac{3}{2}}+D_{k+\frac{3}{2}}\right) v_{k+\frac{3}{2}}+D_{k+\frac{5}{2}} v_{k+\frac{5}{2}}+C_{k+\frac{1}{2}} v_{k+\frac{1}{2}}\right]-\left(f_{k+1}+f_{k+2}\right) v_{k+\frac{3}{2}} \\
\leq & {\left[g_{k+\frac{3}{2}}^{E}+\frac{\left.f_{k+\frac{1}{2}}^{+}+f_{k+\frac{3}{2}}^{+}\right]\left[2 v_{k+\frac{3}{2}}-C_{k+\frac{3}{2}} v_{k+\frac{3}{2}}+C_{k+\frac{1}{2}} v_{k+\frac{1}{2}}\right]-\left(f_{k+1}+f_{k+2}\right) v_{k+\frac{3}{2}}}{4}=\frac{1}{4}\left[\left(4 f_{k+1}+f_{k+2}-f_{s}\right)\left(2 v_{k+\frac{3}{2}}-C_{k+\frac{3}{2}} v_{k+\frac{3}{2}}+C_{k+\frac{1}{2}} v_{k+\frac{1}{2}}\right)-4\left(f_{k+1}+f_{k+2}\right) v_{k+\frac{3}{2}}\right] .\right.}
\end{aligned}
$$

Next, we combine the k-term of $P_{(j \leq k-2)}$, the k-term of $P_{(j \geq k+2)}, P_{k-1}, P_{k}$, and $P_{k+1}$ into one estimate.

$$
\begin{aligned}
T(k):= & \mathrm{k} \text {-term of } P_{(j \leq k-2)}+\mathrm{k} \text {-term of } P_{(j \geq k+2)}+4 P_{k-1}+4 P_{k}+4 P_{k+1} \\
\leq & -f_{k-\frac{1}{2}}^{\prime} v_{k-\frac{1}{2}}^{2}+\left(4 f_{k-1}+f_{k-2}-f_{k}\right) v_{k-\frac{1}{2}} D_{k-\frac{1}{2}} \\
& +f_{k+\frac{3}{2}}^{\prime} v_{k+\frac{3}{2}}^{2}+\left(4 f_{k+2}-f_{k+1}+f_{k+3}\right) v_{k+\frac{3}{2}} C_{k+\frac{3}{2}} \\
& +\left(4 f_{k}+f_{k-1}-f_{s}\right)\left(2 v_{k-\frac{1}{2}}-D_{k-\frac{1}{2}} v_{k-\frac{1}{2}}+D_{k+\frac{1}{2}} v_{k+\frac{1}{2}}\right)-4\left(f_{k-1}+f_{k}\right) v_{k-\frac{1}{2}} \\
& +4 f_{s}\left(2 v_{k+\frac{1}{2}}-C_{k+\frac{1}{2}} v_{k+\frac{1}{2}}-D_{k+\frac{1}{2}} v_{k+\frac{1}{2}}\right)-4\left(f_{k}+f_{k+1}\right) v_{k+\frac{1}{2}} \\
& +\left(4 f_{k+1}-f_{s}+f_{k+2}\right)\left(2 v_{k+\frac{3}{2}}-C_{k+\frac{3}{2}} v_{k+\frac{3}{2}}+C_{k+\frac{1}{2}} v_{k+\frac{1}{2}}\right)-4\left(f_{k+1}+f_{k+2}\right) v_{k+\frac{3}{2}} \\
= & T_{1}(k)+T_{2}(k),
\end{aligned}
$$

where, using $f_{s-\frac{1}{2}}^{\prime} v_{s-\frac{1}{2}} v_{k+\frac{1}{2}}-f_{s-\frac{1}{2}}^{\prime} v_{s-\frac{1}{2}}^{2}=f_{s-\frac{1}{2}}^{\prime} v_{s-\frac{1}{2}} v_{s+\frac{1}{2}}$ and $-f_{s+\frac{1}{2}}^{\prime} v_{s+\frac{1}{2}} v_{k+\frac{1}{2}}+$ $f_{s+\frac{1}{2}}^{\prime} v_{s+\frac{1}{2}}^{2}=-f_{s+\frac{1}{2}}^{\prime} v_{s-\frac{1}{2}} v_{s+\frac{1}{2}}$, we have

$$
\begin{aligned}
T_{1}(k):= & \left(3 f_{k}-f_{k-1}-2 f_{s}\right) v_{k-\frac{1}{2}}+\left(8 f_{s}-4 f_{k}-4 f_{k+1}\right) v_{k+\frac{1}{2}} \\
& +\left(3 f_{k+1}-f_{k+2}-2 f_{s}\right) v_{k+\frac{3}{2}} \\
\leq & f_{s-\frac{1}{2}}^{\prime}\left(v_{s-\frac{1}{2}}-v_{k-\frac{1}{2}}\right)^{2}+f_{s-\frac{1}{2}}^{\prime} v_{s-\frac{1}{2}} v_{s+\frac{1}{2}}+3 f_{s-\frac{1}{2}}^{\prime} v_{s-\frac{1}{2}} v_{k+\frac{1}{2}} \\
& -3 f_{s+\frac{1}{2}}^{\prime} v_{s+\frac{1}{2}} v_{k+\frac{1}{2}}-f_{s+\frac{1}{2}}^{\prime} v_{s-\frac{1}{2}} v_{s+\frac{1}{2}}-f_{s+\frac{1}{2}}^{\prime}\left(v_{s+\frac{1}{2}}-v_{k+\frac{3}{2}}\right)^{2} \\
\leq & 3 f_{s-\frac{1}{2}}^{\prime} v_{s-\frac{1}{2}} v_{k+\frac{1}{2}}-3 f_{s+\frac{1}{2}}^{\prime} v_{s+\frac{1}{2}} v_{k+\frac{1}{2}},
\end{aligned}
$$


and

$$
\begin{aligned}
T_{2}(k):= & \left(-5 f_{k}+3 f_{k-1}+f_{k-2}+f_{s}\right) D_{k-\frac{1}{2}} v_{k-\frac{1}{2}}+\left(f_{k-1}+4 f_{k}-5 f_{s}\right) D_{k+\frac{1}{2}} v_{k+\frac{1}{2}} \\
& +\left(4 f_{k+1}+f_{k+2}-5 f_{s}\right) C_{k+\frac{1}{2}} v_{k+\frac{1}{2}}+\left(-5 f_{k+1}+3 f_{k+2}+f_{k+3}+f_{s}\right) C_{k+\frac{3}{2}} v_{k+\frac{3}{2}} \\
= & {\left[-f_{k-\frac{3}{2}}^{\prime} v_{k-\frac{3}{2}} v_{k-\frac{1}{2}}-4 f_{k-\frac{1}{2}}^{\prime} v_{k-\frac{1}{2}}^{2}+f_{s-\frac{1}{2}}^{\prime} v_{s-\frac{1}{2}} v_{k-\frac{1}{2}}\right] D_{k-\frac{1}{2}} } \\
& +\left[-5 f_{s-\frac{1}{2}}^{\prime} v_{s-\frac{1}{2}} v_{k+\frac{1}{2}}-f_{k-\frac{1}{2}}^{\prime} v_{k-\frac{1}{2}} v_{k+\frac{1}{2}}\right] D_{k+\frac{1}{2}} \\
& +\left[5 f_{s+\frac{1}{2}}^{\prime} v_{s+\frac{1}{2}} v_{k+\frac{1}{2}}+f_{k+\frac{3}{2}}^{\prime} v_{k+\frac{1}{2}} v_{k+\frac{3}{2}}\right] C_{k+\frac{1}{2}} \\
& +\left[f_{k+\frac{5}{2}}^{\prime} v_{k+\frac{5}{2}} v_{k+\frac{3}{2}}+4 f_{k+\frac{3}{2}}^{\prime} v_{k+\frac{3}{2}}^{2}-f_{s+\frac{1}{2}}^{\prime} v_{s+\frac{1}{2}} v_{k+\frac{3}{2}}\right] C_{k+\frac{3}{2}} .
\end{aligned}
$$

Thus,

$$
\begin{aligned}
T(k) \leq & 3 f_{s-\frac{1}{2}}^{\prime} v_{s-\frac{1}{2}} v_{k+\frac{1}{2}}\left(1-\frac{5}{3} D_{k+\frac{1}{2}}\right)-3 f_{s+\frac{1}{2}}^{\prime} v_{s+\frac{1}{2}} v_{k+\frac{1}{2}}\left(1-\frac{5}{3} C_{k+\frac{1}{2}}\right) \\
& +\left[-f_{k-\frac{3}{2}}^{\prime} v_{k-\frac{3}{2}} v_{k-\frac{1}{2}}-4 f_{k-\frac{1}{2}}^{\prime} v_{k-\frac{1}{2}}^{2}\right] D_{k-\frac{1}{2}}-f_{k-\frac{1}{2}}^{\prime} v_{k-\frac{1}{2}} v_{k+\frac{1}{2}} D_{k+\frac{1}{2}} \\
& +f_{k+\frac{3}{2}}^{\prime} v_{k+\frac{1}{2}} v_{k+\frac{3}{2}} C_{k+\frac{1}{2}}+\left[f_{k+\frac{5}{2}}^{\prime} v_{k+\frac{5}{2}} v_{k+\frac{3}{2}}+4 f_{k+\frac{3}{2}}^{\prime} v_{k+\frac{3}{2}}^{2}\right] C_{k+\frac{3}{2}} .
\end{aligned}
$$

Finally, using (3.20), (3.21), (3.22) and (3.23), we have

$$
\begin{aligned}
\text { LHS of }(3.19)= & \sum_{j=I}^{J-1} g_{j+\frac{1}{2}} \bar{v}_{j+\frac{1}{2}}-\sum_{j=I}^{J-1} \int_{v_{j}}^{v_{j+1}} f\left[w ; v_{j}, v_{j+1}\right] d w \\
= & \frac{1}{2}\left(P_{(j \leq k-2)}+P_{k-1}+P_{k}+P_{k+1}+P_{(j \geq k+2)}\right) \\
\leq & \frac{1}{8}\left\{\sum _ { j = I } ^ { k - 2 } \left[\left(-4 f_{j+\frac{1}{2}}^{\prime} v_{j+\frac{1}{2}}^{2}-f_{j-\frac{1}{2}}^{\prime} v_{j-\frac{1}{2}} v_{j+\frac{1}{2}}\right) D_{j+\frac{1}{2}}\right.\right. \\
& +\sum_{j=k+2}^{J-1}\left[\left(f_{j+\frac{3}{2}}^{\prime} v_{j+\frac{1}{2}} v_{j+\frac{3}{2}}+4 f_{j+\frac{1}{2}}^{\prime} v_{j+\frac{1}{2}}^{2}\right) C_{j+\frac{1}{2}}\right. \\
& +3 f_{s-\frac{1}{2}}^{\prime} v_{s-\frac{1}{2}} v_{k+\frac{1}{2}}\left(1-\frac{5}{3} D_{k+\frac{1}{2}}\right)-3 f_{s+\frac{1}{2}}^{\prime} v_{s+\frac{1}{2}} v_{k+\frac{1}{2}}\left(1-\frac{5}{3} C_{k+\frac{1}{2}}\right) \\
& -\left[f_{k-\frac{3}{2}}^{\prime} v_{k-\frac{3}{2}} v_{k-\frac{1}{2}}+4 f_{k-\frac{1}{2}}^{\prime} v_{k-\frac{1}{2}}^{2}\right] D_{k-\frac{1}{2}}-f_{k-\frac{1}{2}}^{\prime} v_{k-\frac{1}{2}} v_{k+\frac{1}{2}} D_{k+\frac{1}{2}} \\
& \left.+f_{k+\frac{3}{2}}^{\prime} v_{k+\frac{1}{2}} v_{k+\frac{3}{2}} C_{k+\frac{1}{2}}+\left[f_{k+\frac{5}{2}}^{\prime} v_{k+\frac{5}{2}} v_{k+\frac{3}{2}}+4 f_{k+\frac{3}{2}}^{\prime} v_{k+\frac{3}{2}}^{2}\right] C_{k+\frac{3}{2}}\right\} .
\end{aligned}
$$

Clearly, it is feasible that

$$
\text { LHS of }(3.19) \leq \frac{1}{2} \sum_{j=I+1}^{J-1} S_{j-1, j, j+1} \leq \sum_{j=I+1}^{J-1} \alpha_{j} S_{j-1, j, j+1},
$$

provided that $\sigma_{\Gamma}$ is sufficiently small. Thus, we have completed the proof of Lemma 3.6.

Acknowledgment. The author likes to express the deepest appreciation of Professor Huanan Yang for his encouragement to start this project few years ago. I am very fortunate to have many opportunities to collaborate with Professor Yang and immensely benefited from his view on the subject. 


\section{REFERENCES}

[1] S. Chakravarthy And S. Osher, High resolution applications of the Osher upwind scheme for the Euler equations, AIAA paper presented at 6th CFD conference 1983.

[2] B. Engquist And S. Osher, Stable and entropy satisfying approximations for transonic flow calculations, Math. Comp., 34 (1980), pp. 45-75.

[3] S. K. Godunov, Finite-difference method for numerical computation of discontinuous solutions of the equations of fluid dynamics, Mat. Sbornik, 47 (1959), pp. 271-306.

[4] A. Harten, High resolution schemes for hyperbolic conservative laws, J. Comput. Phys., 49 (1983), pp. 357-393.

[5] N. Jiang AND H. YAng, On Convergence of Semi-Discrete High Resolution Schemes with van Leer's Flux Limiter for Conservation Laws, Methods and Applications of Analysis, 12:1 (2005), pp. 089-102.

[6] P. Lax and B. Wendroff, Systems of conservation laws, Comm. Pure Appl. Math., 13 (1960), pp. 217-237.

[7] P. LAX, Hyperbolic Systems of Conservation Laws and the Mathematical Theory of Shock Waves, SIAM Regional Conference Series in Applied Mathematics, 11 (1972).

[8] S. Osher AND S. Chakravarthy, High resolution schemes and entropy condition, SIAM J. Numer. Anal., 21 (1984), pp. 955-984.

[9] P. L. RoE, Some contributions to the modeling of discontinuous flows, Lect. Notes Appl. Math., 22 (1985), pp. 163-193.

[10] P. L. RoE, Numerical algorithms for the linear wave equation, Royal Aircraft Establishment Technical Report 81047, 1981.

[11] C.-W. SHU AND S. OsheR, Efficient implementation of essentially non-oscillatory shock capturing schemes, J. Comp. Phys., 83 (1989), pp. 32-45.

[12] P. K. Sweby, High resolution schemes using flux limiters for hyperbolic conservation laws, SIAM J. Numer. Anal., 21 (1984), pp. 995-1011.

[13] E. TADMOR, Convenient total variation diminishing conditions for nonlinear difference schemes, SIAM J. Numer. Anal., 25 (1988), pp. 1002-1014.

[14] B. VAN LEER, Towards the ultimate conservative difference scheme II. Monotonicity and conservation combined in a second order scheme, J. Comput. Phys., 14 (1974), pp. 361-370.

[15] H. YANG, On Wavewise Entropy Inequalities for High-Resolution Schemes I: The SemiDiscrete Case, Math. Comp., 65 (1996), pp. 45-67.

[16] H. YANG, On Wavewise Entropy Inequalities for High Resolution Schemes II: Fully Discrete MUSCL Schemes with Exact Evolution in Small Time, SIAM. J. Numer. Anal., 36:1 (1999), pp. 1-31.

[17] H. YANG AND N. JIANG, On Wavewise Entropy Inequalities for High-Resolution Schemes with Source Terms I: The Semi-Discrete Case, Methods and Applications of Analysis, 10:4 (2003), pp. 487-512. 